\title{
Field emission to control nanometer tip-medium distances in probe storage
}

\author{
Alexander le Fèbre ${ }^{*}$, L. Abelmann, J.C Lodder \\ MESA+ Institute for Nanotechnology, P.O. Box 217, 7500 AE Enschede, Netherlands \\ *Corresponding author: a.j.lefebre@utwente.nl
}

In this work, we present a novel concept for high resolution proximity sensing based on field emission and provide more insight in the vacuum conditions and electronics needed for stable operation. This study is focused on tip-medium distance control, and the aim is to apply this method in a vacuum probe storage device where thousands of field emission probes will be individually positioned at several nanometers above a storage medium [1].

As field emitters we used highly doped $(<0.01 \mathrm{Ohm} \cdot \mathrm{cm})$ single crystal silicon AFM probes. For characterization a UHV STM/AFM microscope was used, by approaching individual probes to a counter-electrode of $\sim 100 \mathrm{~nm}$ TiW sputtered on a silicon wafer sample. To improve the stability of the emission current, the system was operated at $5 \cdot 10^{-9}$ mbar.

The dependence of the field emission current on distance was measured by taking I/V curves (Fig. 1a) for increasing tip-sample distance and fit the corresponding Fowler-Nordheim plots using the procedure described in [2] to obtain the average emission area $\left(5.4 \pm 0.8 \mathrm{~nm}^{2}\right)$ and field enhancement factors.

In Fig. 2 the field enhancement factors are plotted as function of the tip-sample distance. The field enhancement was found to depend non-linearly on the distance due to the changing local geometry. Finite-element analysis was used for a sphere-on-a-stick geometrical model to obtain the distance dependence of the enhancement factor as with $d \gamma=\gamma_{\infty}(r+d) /\left(\gamma_{\infty} r+d\right)$ the tip-sample distance, $r$ the tip radius and $\gamma_{\infty}$ the field enhancement at large distance, determined by the tip sharpness. The fit in Fig. 2 was obtained using this expression for $\gamma_{\infty}=25$ and $r=40 \mathrm{~nm}$, which could be the tip radius of a slightly damaged AFM probe.

Since the emission turn-on voltage is proportional to the tip-sample distance, it can be used in feedback to control the tip position [3]. Displacements were measured as function of voltage by controlling the tip position to maintain a constant current of $3 \mathrm{nA}$ (Fig. 3). The measurement data was fitted using the same model given above. The non-linearity at lower voltages is caused by variations in the electric field enhancement due to the small gap regime, in agreement with the model given above. Fluctuations are caused by instabilities in the emission current. The best fit was obtained by using $\gamma_{\infty}=25$ and $r=5 \mathrm{~nm}$.

To test the field emission control method for scanning on a storage medium, a sample of $35 \mathrm{~nm}$ high SiN nanodots with metal coating was prepared by laser interference lithography (LIL), see Fig. 4. Fig. 5 shows $1 \times 1 \mu \mathrm{m}$ scans on this sample at a constant current of $0.3 \mathrm{nA}$ using $0.5 \mathrm{~V}, 5.0 \mathrm{~V}$ and $50.0 \mathrm{~V}$ bias voltage respectively. Increasing the bias voltage results in a loss of resolution, caused by the increase in tip-sample distance. Although the noise level increases due to instabilities in the field emission current, the signal is still sufficient to be able to track the dots. The current stability may be improved by changing the probe coatings, improving the vacuum conditions and thermal conditioning of probes and sample.

Our measurements confirm that field emission can be applied to control the spacing between tip and medium, with sufficient resolution and current stability for probe storage applications. Next to tip-medium distance control, also the cantilever resonance frequency was detected from the field emission current [4], which enables an integrated method for reading data in magnetic probe storage. 

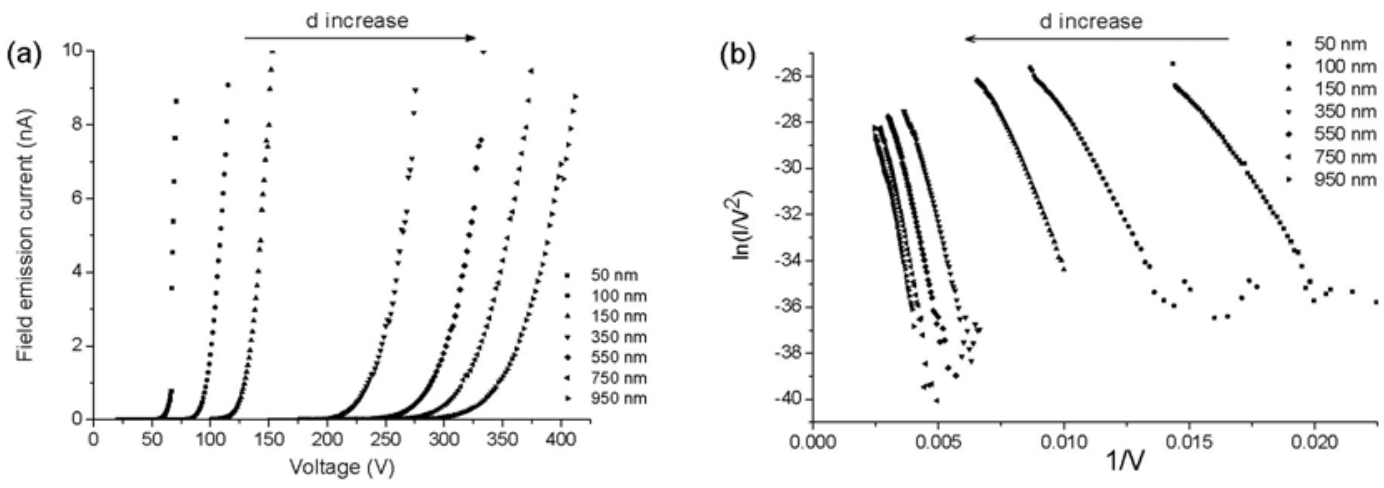

Fig.1. Distance dependence of field emission in (a) I/V characteristics and (b) corresponding FowlerNordheim plots, measured for gaps from $50 \mathrm{~nm}$ to $950 \mathrm{~nm}$ between standard single-crystal silicon AFM probe and silicon sample coated with $100 \mathrm{~nm}$ TiW.

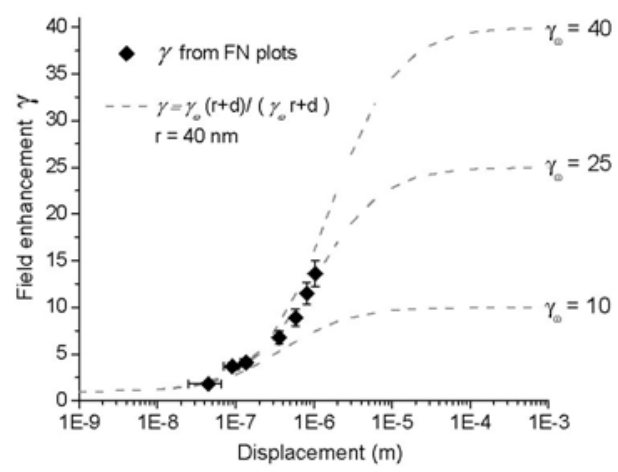

Fig.2. Field enhancement factors determined by fitting the Fowler-Nordheim plots (Fig. 1b), plotted as function of electrode distance. Lines are fits of the field enhancement model with $r=40 \mathrm{~nm}$.

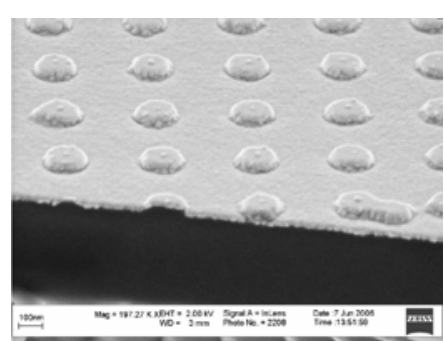

Fig. 4. SEM image of the LIL patterned sample (dot height $35 \mathrm{~nm}$, periodicity $365 \mathrm{~nm}$ ), coated with $\mathrm{Cr}(5) \operatorname{Pt}(15)$.

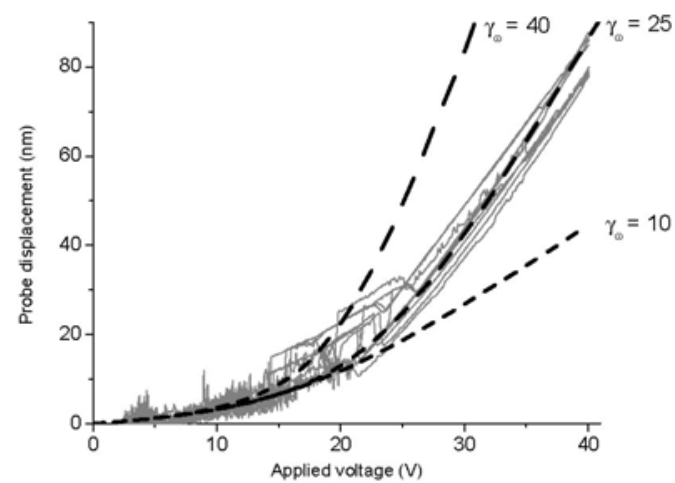

Fig.3. Displacement measured as function of applied voltage (constant current $3 \mathrm{nA}$ ). Fits determined by field enhancement model using $r=5 \mathrm{~nm}$. Note that this measurement is on a tip different from Fig. 2.

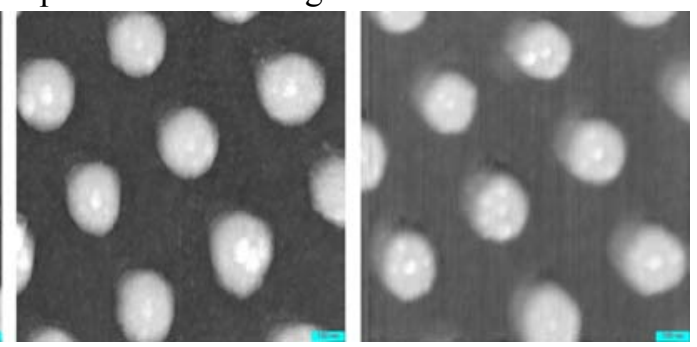

Fig.5. Scanning the emission probe constant current ( $3 \mathrm{nA}$ ) during position feedback gives a height image of the LIL sample. The bias voltage is changed to $0.5 \mathrm{~V}$ (left), $5.0 \mathrm{~V}$ (middle) and $50.0 \mathrm{~V}$ (right) to increase the tip-sample distance, resulting in a loss of resolution.

[1] le Febre, A.J. et al, J. Phys.: Conf. Ser. 61, 673, (2007).

[2] Forbes, R.G., Ultramicroscopy 79, 11, (1999).

[3] Young, R.D., Rev Sci Instr 37, 275, (1966).

[4] Yang, C.K. et al, "Field Emission for Resonance Sensing in MEMS/NEMS", accepted for this conference (O-34). 